\title{
Los receptores epidérmicos humanos en el cáncer gástrico: alteraciones moleculares y su papel como diana terapéutica
}

\section{Andrea Rebeca Bustos-Carpinteyro ${ }^{1,2}$, María Teresa Magaña-Torres', Juan Ramón González-García ${ }^{1}$, Juan Heriberto Torres-Jasso ${ }^{3}$ y Josefina Yoaly Sánchez-López ${ }^{1}$}

${ }^{1}$ División de Genética, Centro de Investigación Biomédica de Occidente, Instituto Mexicano del Seguro Social; '2Doctorado en Genética Humana, Centro Universitario de Ciencias de la Salud, Universidad de Guadalajara. Guadalajara, Jal.; ${ }^{3}$ Departamento de Ciencias Biológicas, División de Ciencias Biológicas y de la Salud, Centro Universitario de la Costa, Universidad de Guadalajara, Delegación Ixtapa, Puerto Vallarta, Jal. México

\begin{abstract}
Resumen
El cáncer gástrico (CG) es la tercera causa de muerte por cáncer en todo el mundo; tanto los factores ambientales como los genéticos están implicados en la etiología de esta neoplasia. La vía de receptores epidérmicos humanos (HER, human epidermal receptors) es esencial para la proliferación y la diferenciación de las células normales, pero también está involucrada en el crecimiento de células cancerosas. En este trabajo investigamos las alteraciones moleculares en los genes que codifican para los receptores HER reportadas en CG, así como su papel como dianas terapéuticas. Hemos revisado la literatura en relación con la sobreexpresión y la amplificación de los receptores HER, las mutaciones en los genes que codifican para estos receptores en los tumores gástricos y las terapias anti-HER que se han probado para el tratamiento del CG. Se encontró que la sobreexpresión de HER está reportada en el 12 al 87\% de los casos de CG; la amplificación hasta en el 67\% de los casos, y 90 mutaciones somáticas se han reportado en los genes ERBB en pacientes con CG. El único fármaco anti-HER aprobado para usarse en combinación con quimioterapia en el CG avanzado es el trastuzumab, pero se están investigando otras terapias dirigidas. En conclusión, el papel de la familia HER como diana terapéutica en el CG no ha mostrado mejoras significativas en los últimos años, y por lo tanto se requieren más estudios para encontrar mejores opciones para el tratamiento del CG.
\end{abstract}

PALABRAS CLAVE: Proliferación celular. Amplificación génica. Genes ERBB. Transducción de señales. Cáncer gástrico.

\begin{abstract}
Gastric cancer (GC) is the third leading cause of cancer death worldwide; both environmental and genetic factors are involved in the etiology of this neoplasia. The human epidermal receptor (HER) pathway is essential for proliferation and differentiation of normal cells; but it is also implicated in the growth of cancer cells. In this work we investigate the molecular alterations in genes that encodes for HER receptors reported in GC, as well the role as therapeutic targets. We reviewed the literature reported to date regarding overexpression of HER-receptors, amplification and somatic mutations in ERBB genes occurred in gastric tumors, as well as the anti-HER therapies tested for treatment of GC. In GC, the overexpression of HER family is reported in a range of $12-87 \%$ of cases; up to $67 \%$ of cases with amplification, and 90 somatic mutations in ERBB genes. The only drug anti-HER approved for using combined with chemotherapy, in treatment of patients with advanced GC is trastuzumab; however, other targeted therapies are being investigated. The role of the HER family as a therapeutic target has not shown significant improvements in recent years; hence, further studies are required to find better options for treatment of GC.
\end{abstract}

KEY WORDS: Cell proliferation. Gene amplification. ERBB genes. Signal transduction. Stomach neoplasms.

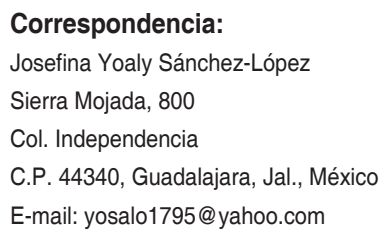

Fecha de recepción en versión modificada: 08-11-2016

Fecha de aceptación: 01-12-2016

DOI://dx.doi.org/10.24875/GMM.17002748
Gac Med Mex. 2017;153:830-840

Contents available at PubMed www.gacetamedicademexico.com 


\section{Introducción}

En todo el mundo, el cáncer gástrico (CG) es el quinto tipo de cáncer más común y la tercera causa de muerte por cáncer1. Varias vías de señalización relacionadas con el cáncer se activan en el CG. La vía del receptor epidérmico humano (HER, human epidermal receptor) es una de las más importantes de las vías de transducción de señales que están implicadas en el crecimiento del tumor y la diferenciación en diferentes tipos de cáncer, incluyendo el $\mathrm{CG}^{2}$.

La familia HER es un grupo de cuatro glucoproteínas transmembrana tirosina cinasa: HER1 (EGFR o ERBB1), HER2 (NEU o p185 ${ }^{\text {HER2 }}$ ), HER3 y HER4. La familia HER desempeña un papel importante en la proliferación celular, la diferenciación y la migración en las células normales, así como en la invasión, la angiogénesis, la metástasis y la apoptosis en células tumorales $^{3}$ (Fig. 1).

Se reconocen tres vías en la señalización HER: fosfatidilinositol 3-cinasa (PI3K)/AKT (PKB), RAS/ RAF/MEK/ERK1/2 y fosfolipasa C (PLCg) ${ }^{3,4}$. La vía PI3K/AKT desempeña un papel importante en la mediación de la supervivencia celular, mientras que RAS/ERK1/2 y las vías PLCg participan en la proliferación celular.

\section{Alteraciones moleculares en la familia HER}

\section{Sobreexpresión}

La inmunorreactividad para la sobreexpresión de HER se categoriza como 0 ( $<10 \%$ de células con una reactividad membranosa débil), $1+(>10 \%$ de las células con una reactividad membranosa parcial y débil), $2+$ (> 10\% de las células tumorales con una reactividad leve a moderada en la membrana basolateral o completa) $03+$ (> 10\% de células que muestran reactividad moderada a fuerte en la membrana basolateral o completa). Los tumores gástricos con una clasificación IHC 3+ se consideran HER positivos (sobreexpresión); en contraste, las muestras con IHC 2+ deben ser evaluadas por hibridación in situ para establecer el estado de expresión de HER ${ }^{5}$.

La sobreexpresión de HER1 en los tumores gástricos varía ampliamente $\left(2-63 \%\right.$ de los casos) ${ }^{6-8}$. La sobreexpresión de HER1 se localiza principalmente en el citoplasma ( $45 \%$ de los casos), mientras que solo el $9 \%$ exhibe expresión membranosa ${ }^{7-9}$ y no se encuentra en el núcleo. La sobreexpresión se ha relacionado con la edad avanzada, un curso más agresivo de la enfermedad (poca diferenciación, tipo difuso y capacidades infiltrativas), una localización proximal y una fase avanzada de la enfermedad ${ }^{6}$; sin embargo, el papel pronóstico de la sobreexpresión de HER1 en el GC no está clara, porque en algunos estudios la positividad de HER1 se asoció significativamente con un mal pronostico ${ }^{10,11}$, mientras que otros autores no pudieron demostrar una correlación significativa entre estos factores ${ }^{7,12,13}$.

La sobreexpresión de HER2 también varía ampliamente en el CG (del 4.4 al $53.4 \%$ de los casos) ${ }^{14}$. La sobreexpresión es más común en el citoplasma (62\%) que en la membrana (17\%), y también es más frecuente en el subtipo intestinal (32\%) que en el difuso $(21 \%)^{6,14}$, y en los pacientes de edad avanzada 7 .

HER3 está sobreexpresado en el $87 \%$ de los tumores gástricos ${ }^{15}$, y es más común en el citoplasma $(64 \%)$ que en el núcleo (34\%) o la membrana $(2 \%)^{6,7}$, aunque Choi, et al. ${ }^{15}$ observaron que HER3 se encuentra principalmente en el núcleo. Para algunos autores, la sobreexpresión de HER3 se relaciona con el subtipo intestinal de $\mathrm{CG}^{6,7,16}$, mientras que para otros se relaciona con el subtipo difuso ${ }^{17}$. Por otra parte, también se ha descrito la falta de asociación con algún subtipo de $\mathrm{CG}^{18,19}$. Además, la sobreexpresión se asocia con tumores bien y moderadamente diferenciados, edad avanzada, mayor invasión tumoral, afectación ganglionar, metástasis tumoral y pobre tasa de sobrevida ${ }^{16,7,18}$. La expresión nuclear de HER3 en el CG se asocia con invasión vascular y linfática, y con baja supervivencia (HER3+ 24-28 meses vs. HER3- 45-47 meses) $)^{6,16}$.

La expresión de HER4 parece ser mayor en los tejidos gástricos tumorales que en la mucosa gástrica adyacente ${ }^{6}$. La sobreexpresión es citoplásmica en el $23-24 \%$ de los casos, membranal en el $15-18 \%$ y nuclear en el $2 \% 6,7$. La sobreexpresión de HER4 se asocia con características tumorales de buen pronóstico, tales como subtipo intestinal, diferenciación buena o moderada, estadios tumorales $\mathrm{T} 1 / \mathrm{T} 2$, no metástasis en ganglios linfáticos y no invasión vascular, linfática ni perineural ${ }^{6}$. Esto es concordante con el papel de HER4 en la diferenciación y la respuesta antiproliferativa ${ }^{20}$. A pesar de su supuesta implicación en un mejor pronóstico en los pacientes con CG, algunos informes han demostrado que la sobreexpresión de HER4 está asociada con etapas avanzadas de la enfermedad ${ }^{16} \mathrm{y}$ con la presencia de células en anillo de sello ${ }^{21}$. 


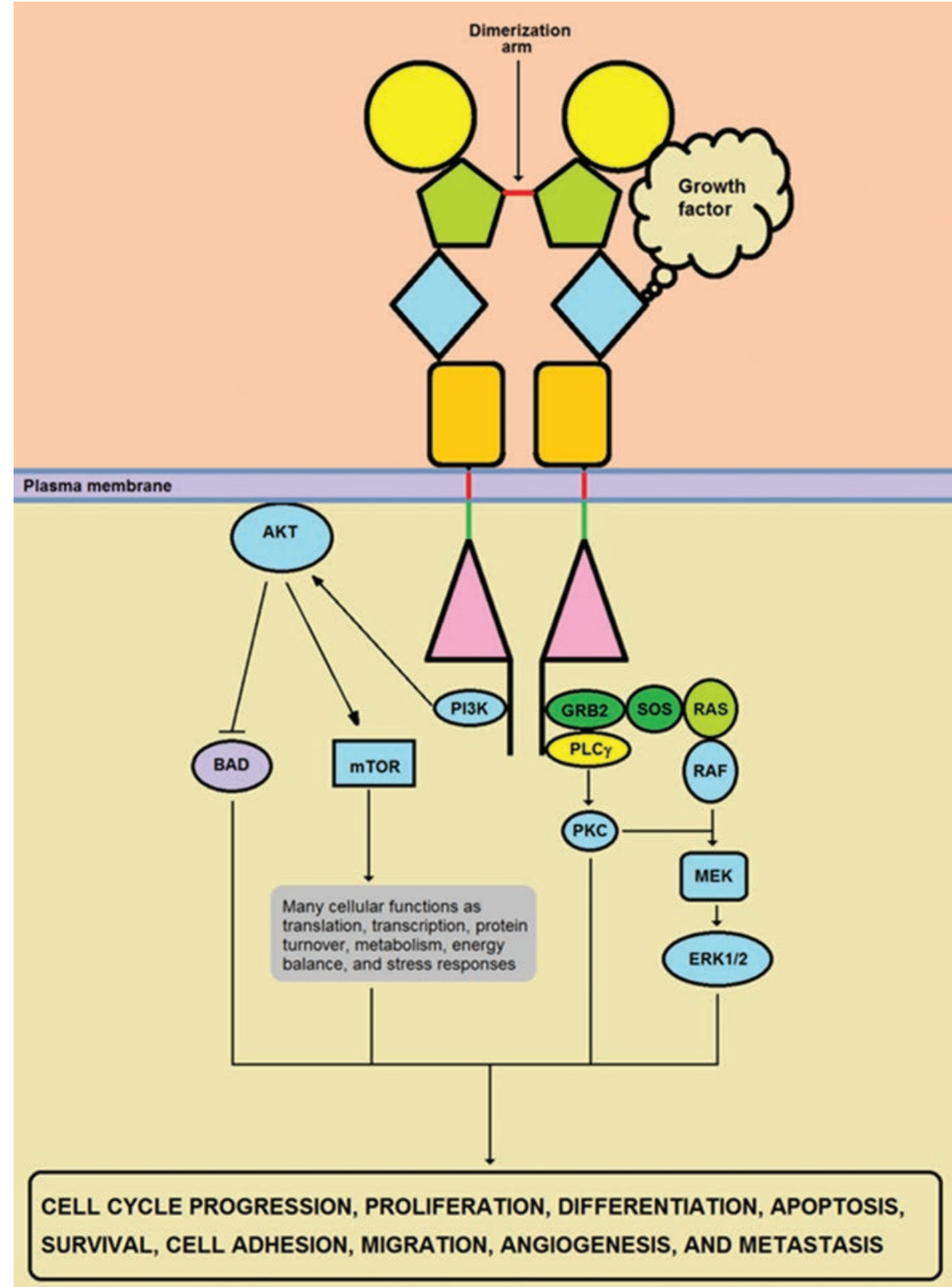

Figura 1. Esquema general del proceso de dimerización y las principales vías de señalización en la familia de receptores HER. La unión al ligando afecta a los dominios I y III (excepto para HER2).

\section{Amplificación}

La amplificación de los genes ERBB se define como positiva cuando el radio de señales HER: CEP es $\geq 2$. La polisomía de cromosomas se define como $\geq 3$ señales CEP en promedio por célula. En el análisis basado en hibridación in situ fluorescente o cromogénica se utiliza un sistema de coloración dual, con la sonda para alguno de los genes EGFR, ERBB2, $E R B B 3$ o ERBB4 y con la respectiva sonda cromosómica centromérica (CEP).
La amplificación del gen EGFR se ha identificado en el 2 al $29 \%^{22-25}$ de los casos de CG, y la polisomía del cromosoma 7 en el 3 al 10\% de los casos. La amplificación de ERBB2 se informa en el 8 al 22,1\% de los tumores gástricos ${ }^{5,6,22,26-30}$ y se asocia con subtipo intestinal, tumores gástricos bien o moderadamente diferenciados, y una sobrevida reducida (17 meses para HER2+ vs. 40 meses para HER2-)6. La amplificación de ERBB3 en el $C G$ aún no ha sido observada ${ }^{6}$. La amplificación de ERBB4 en el CG fue informada por Nielsen, et al. ${ }^{20}$ en el $67 \%$ de los casos, 
Tabla 1. Mutaciones somáticas en los genes de la familia ERBB reportadas en cáncer gástrico

\begin{tabular}{|c|c|c|c|c|}
\hline \multirow{2}{*}{$\frac{\operatorname{EGFR}(\mathrm{n}=19)}{{ }^{\circ K} 189 \mathrm{~T}^{42}}$} & \multicolumn{2}{|c|}{ HER2 $(\mathrm{n}=34)$} & \multirow{2}{*}{$\begin{array}{c}\text { HER3 }(\mathbf{n}=\mathbf{2 0}) \\
\text { f. } \mathrm{V} 104 \mathrm{M}^{44}\end{array}$} & \multirow{2}{*}{$\frac{\text { HER4 }(\mathrm{n}=\mathbf{1 7})}{{ }^{n} \mathrm{~V} 59 \mathrm{D}^{42}}$} \\
\hline & e,j|101V43 & ${ }^{\mathrm{d} D} \mathrm{D} 69 \mathrm{H}^{34}$ & & \\
\hline cW200X ${ }^{42}$ & еT216P 43 & ${ }^{d} G 776 S^{35}$ & 'Y111C 44 & h,íL91P43 \\
\hline${ }^{\mathrm{a} C} \mathrm{C} 232 \mathrm{~S}^{43}$ & ${ }^{e} D 234 A^{43}$ & ${ }^{d} V 777 L^{34}$ & ${ }^{\mathrm{f}} \mathrm{R} 135 \mathrm{~L}^{44}$ & h.iv2261 ${ }^{43}$ \\
\hline aV308 $A^{43}$ & ${ }^{e} \mathrm{C} 235 S^{43}$ & ${ }^{\mathrm{d}} \mathrm{Q799P}{ }^{34}$ & ${ }^{9} \mathrm{~A} 172 \mathrm{P}^{43}$ & ${ }^{n} \mathrm{~K} 324 N^{43}$ \\
\hline${ }^{\mathrm{a}} 309 \mathrm{G} \mathrm{G}^{43}$ & e, D277Y Y3 & dV842/33 & f.jA232V ${ }^{44}$ & h,il $\mid 329 M^{43}$ \\
\hline${ }^{\mathrm{a}} \mathrm{C} 31 \mathrm{H}_{1 \mathrm{G}}{ }^{43}$ & eV296L ${ }^{43}$ & 'T862A ${ }^{42}$ & f.jp262H ${ }^{44}$ & $\mathrm{~h}_{\mathrm{i}} \mathrm{A} 380 \mathrm{E}^{43}$ \\
\hline aV505G $G^{43}$ & e. $V 308 G^{43}$ & ${ }^{d} L_{869 Q}{ }^{34}$ & 'G284R ${ }^{42}$ & h. $\mathrm{H} 505 \mathrm{P}^{43}$ \\
\hline${ }^{\mathrm{C}} \mathrm{T} 638 \mathrm{M}^{42}$ & dS $310 F^{42}$ & ${ }^{e} \mathrm{E} 914 G^{43}$ & fD297Y43 & nW513G ${ }^{43}$ \\
\hline${ }^{b} \mathrm{C} 646 X^{43}$ & ${ }^{e} \mathrm{C} 331 \mathrm{G}^{43}$ & eL994W"3 & ${ }^{\mathrm{f}} \mathrm{E} 332 \mathrm{~K}^{42}$ & ${ }^{n} A 773 S^{37}$ \\
\hline${ }^{b} N 647 D^{43}$ & ${ }^{e} \mathrm{D} 417 \mathrm{H}^{43}$ & e:G995A 43 & 'M406T ${ }^{44}$ & ${ }^{h} L 798 R^{42}$ \\
\hline${ }^{\mathrm{c}} \mathrm{A} 767 \mathrm{~V}^{23}$ & ${ }^{e} \mathrm{D} 417 \mathrm{~A}^{43}$ & e: $\mathrm{Q} 1206 \mathrm{H}^{43}$ & ${ }^{\mathrm{t}} \mathrm{R} 453 \mathrm{H}^{44}$ & h,iK966T ${ }^{43}$ \\
\hline${ }^{\circ} \mathrm{C} 781 S^{32}$ & e.iT526P ${ }^{43}$ & ${ }^{e} \mathrm{~A} 1216 \mathrm{D}^{43}$ & ${ }^{\mathrm{f}} \mathrm{R} 475 \mathrm{~W}^{42}$ & ${ }^{\mathrm{h}} \mathrm{F} 974 \mathrm{~V}^{43}$ \\
\hline cY801C & e, N530T 43 & ${ }^{e} \mathrm{~F} 1217 S^{43}$ & ${ }^{f} K 498 I^{44}$ & ${ }^{h} \mathrm{~F} 1064 L^{42}$ \\
\hline cN842D ${ }^{23}$ & e, L637V43 & eF1217C 43 & V654G $G^{43}$ & ${ }^{h} E 1199 K^{42}$ \\
\hline cV843| & ${ }^{e} \mathrm{C} 642 \mathrm{~S}^{43}$ & & ${ }_{\mathrm{f}, \mathrm{j}} \mathrm{Q} 809 \mathrm{R}^{44}$ & ${ }^{h} E 1201 X^{43}$ \\
\hline${ }^{c} \mathrm{~L}^{2} 8 \mathrm{R}^{31}$ & ${ }^{\mathrm{d}} \mathrm{R} 678 \mathrm{Q}^{42}$ & & ${ }^{\mathrm{f} K 926 \mathrm{R}^{43}}$ & ${ }^{\mathrm{n}} \mathrm{A} 1233 \mathrm{G} \mathrm{G}^{42}$ \\
\hline${ }^{c} G 863 D^{23}$ & $\mathrm{~d} K 724 \mathrm{~N}^{34}$ & & ${ }^{\mathrm{f}} \mathrm{E} 928 \mathrm{G}^{36}$ & ${ }^{\mathrm{h}} \mathrm{R} 1250 \mathrm{Q}^{42}$ \\
\hline${ }^{c} Y 1016 S^{43}$ & dT733| ${ }^{34}$ & & fL930W 43 & \\
\hline c. $V 1109 D^{43}$ & $\mathrm{~d} \mid 740 \mathrm{~T}^{42}$ & & ${ }^{\mathrm{t} R} 1089 \mathrm{~W}^{44}$ & \\
\hline & $\mathrm{d}, \mathrm{e}$ L755S $\mathrm{S}^{34,43}$ & & ${ }^{\dagger} 1218 \mathrm{delE}^{36}$ & \\
\hline
\end{tabular}

Número de secuencias usadas para nombrar cada mutación: aNM_201282 (628 aa); bNM_201284 (705 aa); ‘NM_005228 (1210 aa); 'NM_004448 (1255 aa); elas mutaciones descritas por Holbrook, et al. 43 considerando la isoforma 1225 aa (NM_001005862) fueron nombradas por nosotros de acuerdo con la isoforma 1255 aa (NM_004448); 'NM_001982 (1342 aa); 9NM_001005915 (183 aa); ' NM_005235 (1308 aa); imutaciones con un SIFT score > 0.05: el cambio de aminoácido posiblemente no afecta de manera significativa la función de la proteína; 'evidencia de actividad oncogénica con HER2 tipo silvestre.

pero Begnami, et al. ${ }^{6}$ no observaron amplificación del gen ERBB4, lo que denota la heterogeneidad entre los tumores gástricos.

\section{Mutaciones en genes que codifican la familia HER}

El análisis de mutaciones en los genes de la familia HER se ha llevado a cabo con mayor frecuencia en el dominio tirosina cinasa que en los otros dominios $^{23,31-37}$. Las mutaciones no se han detectado en todos los estudios, en parte por el tipo de tumor analizado (difuso o intestinal), por el estadio tumoral e incluso por las diferencias étnicas ${ }^{33,34,38-42}$.

En el GC se han reportado al menos 90 mutaciones somáticas no sinónimas en los genes $E R B B$, de las cuales 86 son de sentido erróneo (missense), tres son mutaciones sin sentido (nonsense) y una es la deleción de un codón completo (Tabla 1).

La distribución de estas mutaciones en cada gen ERBB es: 19 en EGFR, 34 en ERBB2, 20 en ERBB3 y 17 en ERBB4. Estas mutaciones afectan a todos los dominios del receptor, aunque con mayor frecuencia al dominio cinasa (27/90, 30.0\%) (Fig. 2). Holbrook, et al. ${ }^{43}$ identificaron un 50\% (44/88) de estas mutaciones y determinaron su posible impacto funcional; además, predijeron que 28 de ellas pueden tener un papel importante en el desarrollo o la progresión del cáncer (Tabla 1).

\section{Mutaciones en el gen EGFR}

Las mutaciones en el gen EGFR en el GC son raras o ausentes, de acuerdo con la mayoría de los 


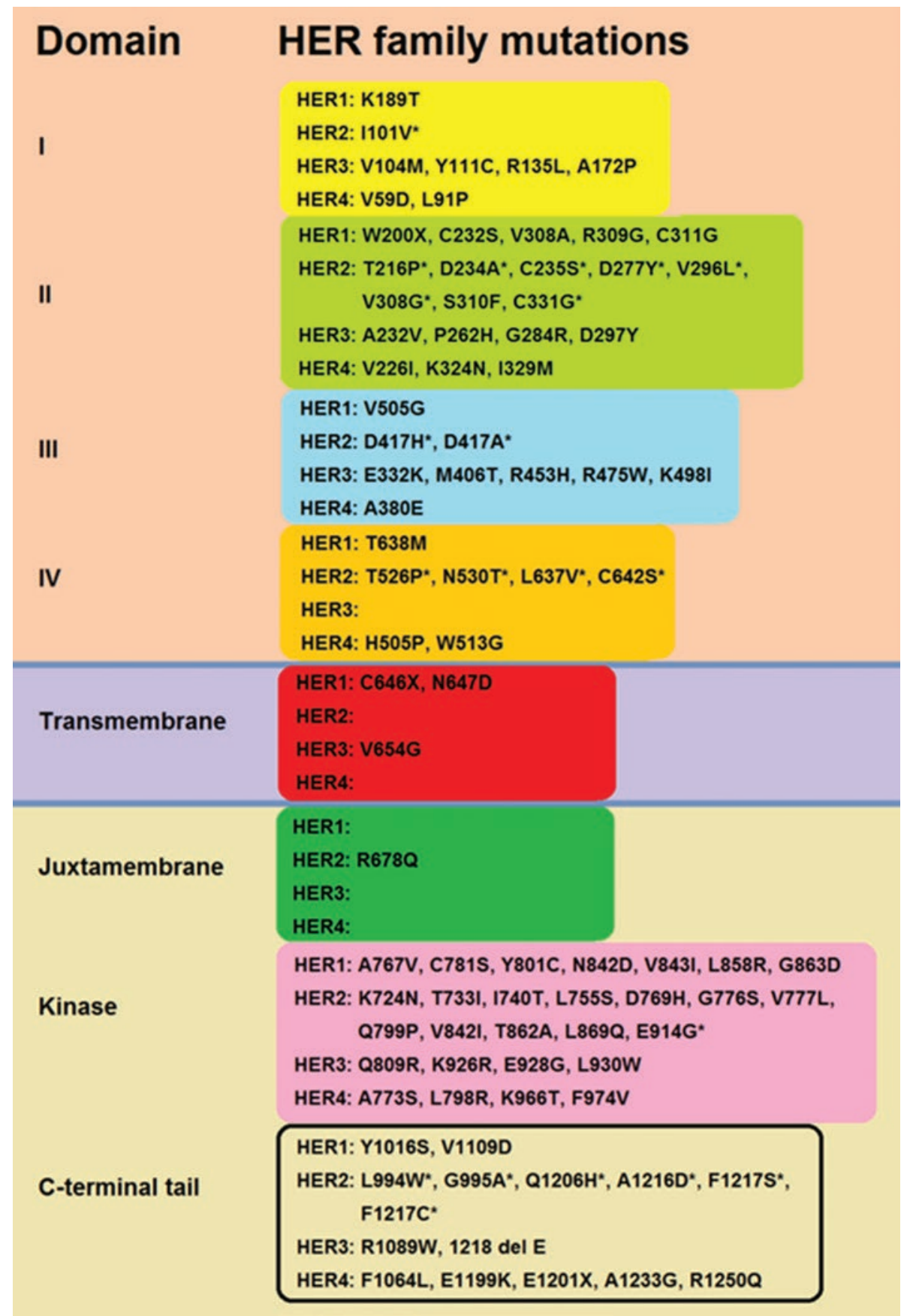

Figura 2. Localización de las mutaciones reportadas que afectan a la familia de receptores ERBB-HER en el cáncer gástrico. Las mutaciones marcadas con asterisco fueron descritas por Holbrook, et al. ${ }^{43}$ considerando la isoforma 1225 aminoácidos (NM_001005862); las reubicamos en la figura de acuerdo con la isoforma 1255 aminoácidos (NM_004448).

informes. Su incidencia varía en diferentes estudios, desde el $0 \%{ }^{33,38-41}$, el $5.1 \%^{31,32}$ y hasta el $30 \%{ }^{43}$. Se han observado 19 mutaciones somáticas diferentes (17 de sentido erróneo y dos sin sentido), produciéndose principalmente en el dominio de la tirosina cinasa (7/19) y en el dominio II (5/19) (Fig. 2 y Tabla 1). Se observaron algunas mutaciones somáticas recurrentes en dos o más casos de CG: V308A, V505G y
Y1016S. Moutinho, et al. ${ }^{23}$ realizaron un análisis de la correlación entre las alteraciones en el gen EGFR y las características clínicas, y sugirieron que EGFR está implicado en la regulación del tamaño del tumor y la progresión. Las mutaciones activantes en EGFR no son frecuentes en el CG, pero otras alteraciones, como mutaciones o variaciones del número de copia, se encuentran implicadas en un aumento del tamaño 
de los tumores difusos; además, se ha sugerido que alteraciones en este gen confieren un comportamiento invasivo en las células neoplásicas.

\section{Mutaciones del gen ERBB2}

Se han realizado pocos estudios para identificar mutaciones somáticas de ERBB2 en el CG en comparación con los cánceres de pulmón y de mama. La tasa de mutación de ERBB2 en el CG varía del $2 \%^{33-35}$ al $60 \%{ }^{43}$; sin embargo, de las 30 mutaciones detectadas por Holbrook, et al. ${ }^{43}, 14$ parecen ser poco importantes para el desarrollo o la progresión del cáncer, disminuyendo así la frecuencia de mutaciones de ERBB2 con impacto tumoral al $32 \%$. Hasta la fecha se han descrito 34 variantes de ERBB2 diferentes de sentido erróneo, de las cuales 13 se detectaron en el dominio tirosina cinasa ${ }^{33-35,42,43}$ (Fig. 2) y en CG avanzado ${ }^{34}$. De manera similar a la observada para el EGFR, la mayoría de las mutaciones en ERBB2 son heterogéneas en diferentes tipos de tumores. Muy pocas mutaciones son recurrentes, tales como D204A, L755S, R678Q y V777L, entre otras ${ }^{34,42,43}$. Algunas mutaciones se observan en varios pacientes con el mismo tipo de cáncer y otras en pacientes con diferentes tipos de cáncer. Por otra parte, el mismo codón es cambiado por distintos aminoácidos, sugiriendo la presencia de puntos susceptibles de mutación (hot spots); por ejemplo, L755S/W/P, D769H/Y, G776S/L/V y V777L/M³4,35,45-47. La mutación L755S (denominada L725S por Holbrook, et al. ${ }^{43}$ ) es una de las mutaciones más frecuentemente observadas en varios tipos de cáncer. Las mutaciones somáticas D769H, D769Y, V777L y V842I son mutaciones activantes que pueden, por sí mismas, promover la proliferación celular ${ }^{45}$.

\section{Mutaciones del gen ERBB3}

La frecuencia de mutaciones en el gen ERBB3 en el $C G$ varía del $0.6 \%{ }^{48}$ al $13 \%{ }^{44}$. Actualmente ya se han identificado al menos 20 mutaciones somáticas en ERBB3 (19 de sentido erróneo y una deleción de un codón completo). A diferencia de lo observado para EGFR y ERBB2, la mayoría de las mutaciones se producen dentro del dominio III, el cual participa en la unión al ligando ${ }^{36,42,43,44}$ (Fig. 2). Jaiswal, et al. ${ }^{44}$ analizaron el impacto funcional de cuatro mutaciones del gen ERBB3 (V104M, A232V, P262H0 y Q809R) y encontraron evidencia de potencial oncogénico en todas ellas. Por otra parte, Holbrook, et al. ${ }^{43}$ analizaron cinco mutaciones del gen ERBB3 (A172P, D297Y,
V654G, K926R y L930W) y encontraron una posible relevancia clínica para el CG. La mayoría de estas mutaciones tienen importancia funcional, ya que afectan las interacciones que son necesarias para la dimerización o la unión al ligando ${ }^{44}$.

\section{Mutaciones en el gen ERBB4}

La frecuencia de las mutaciones del gen ERBB4 en el CG varía del $0.6 \%{ }^{37}$ al $28 \%{ }^{43}$. Existen por lo menos 17 diferentes mutaciones somáticas reportadas en el CG (16 son de sentido erróneo y una sin sentido). Las mutaciones se distribuyen por todo el gen; sin embargo, se observa una ligera acumulación en el dominio C-terminal (Fig. 2 y Tabla 1). Aunque no se ha realizado una caracterización funcional de las mutaciones del gen ERBB4 en el CG, Holbrook, et al..$^{43}$ calcularon el potencial de 10 variantes y encontraron que al menos cuatro mutaciones (K324N, W513G, F974V y E1201X) pueden ser clínicamente importantes para el desarrollo del cáncer.

\section{Terapias dirigidas contra la familia de receptores HER}

Hay dos clases de terapias anti-HER: los anticuerpos monoclonales y los inhibidores de moléculas pequeñas de tirosina cinasa. La monoterapia anti-HER para el CG es menos tóxica, pero también menos eficiente, por lo que se utiliza más comúnmente la combinación de agentes dirigidos contra HER con los agentes quimioterapéuticos tradicionales.

\section{Anticuerpos monoclonales}

\section{Terapias anti-HER1}

El cetuximab es una inmunoglobulina G1 (IgG1) tipo anticuerpo monoclonal quimérico que se une al dominio extracelular del HER1 e inhibe competitivamente la unión del ligando EGF y de otros ligandos, así como la autofosforilación tirosina cinasa inducida por el ligando. El cetuximab también induce la internalización, la subregulación y la degradación de $E G F R^{49}$. Fue el primer anticuerpo anti-EGFR aprobado por la Food and Drug Administration (FDA) de los EE.UU. en 2004 para el tratamiento de carcinomas de células escamosas y cáncer colorrectal avanzado de tipo KRAS silvestre ${ }^{50,51}$. Es la terapia anti-HER1 más investigada en el $\mathrm{CG}^{52-54}$. No ha tenido un impacto significativo en la sobrevida (3.1-16.6 meses) ni en la 
Gaceta Médica de México. 2017;153

Tabla 2. Terapias HER dirigidas para pacientes con cáncer gástrico (estudios con resultados publicados)

\begin{tabular}{|c|c|c|c|c|c|}
\hline Fármaco & Diana & Combinado con quimioterapia & SLP (meses) & SP (meses) & Referencia \\
\hline \multicolumn{6}{|c|}{ Anticuerpos monoclonales } \\
\hline \multirow[t]{14}{*}{ Cetuximab } & HER1 & Irinotecán, leucovorina, 5FU† & $8-9$ & $16-16.6$ & Pinto, et al. ${ }^{55}$ \\
\hline & & & & & Kanzleer, et al. ${ }^{56}$ \\
\hline & & & & & Moehler, et al. ${ }^{57}$ \\
\hline & & Cisplatino, leucovorina, $5 \mathrm{FU}^{+}$ & 11 & 14.5 & Yeh, et al..$^{58}$ \\
\hline & & Oxaliplatino, capecitabina $^{\dagger}$ & 6.5 & 11.8 & Kim, et al. ${ }^{52}$ \\
\hline & & Oxaliplatino, leucovorina, $5 \mathrm{FU}^{+}$ & 5.5 & 9.9 & Han, et al. ${ }^{59}$ \\
\hline & & Oxaliplatino, docetaxel ${ }^{\dagger}$ & 5.1 & 9.4 & Richards, et al. ${ }^{60}$ \\
\hline & & Cisplatino, docetaxe ${ }^{\dagger}$ & 5 & 9 & Pinto, et al..$^{61}$ \\
\hline & & Capecitabina, cisplatino ${ }^{\dagger}$ & 4.4 & 9.4 & Lordick, et al. ${ }^{62}$ \\
\hline & & Irinotecán, leucovorina, 5FUł & 4.9 & 8.1 & $\mathrm{Li}$, et al. ${ }^{63}$ \\
\hline & & Irinotecán ${ }^{\ddagger}$ & 2.8 & 6.1 & Schonnemann, et al. ${ }^{64}$ \\
\hline & & $\begin{array}{l}\text { Irinotecán }{ }^{\ddagger} \\
\text { Irinotecán, leucovorina, 5FU } \\
\text { Irinotecán, cisplatino } \\
\text { Oxaliplatino, leucovorina, 5FU } \\
\text { Docetaxel, cisplatino } \neq\end{array}$ & 1.7 & 3.2 & Park, et al. ${ }^{65}$ \\
\hline & & Docetaxel $\left.\right|^{\ddagger}$ & 2.1 & 5.4 & Tebbutt, et al. ${ }^{66}$ \\
\hline & & Ninguno $0^{\ddagger}$ & 1.6 & 3.1 & Chan, et al. ${ }^{67}$ \\
\hline Panitumumab & HER1 & 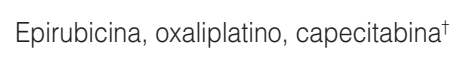 & 6 & 8.8 & Waddell, et al. ${ }^{68}$ \\
\hline Matuzumab & HER1 & 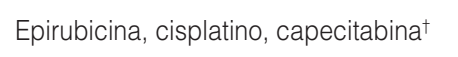 & 4.8 & 9.4 & Rao, et al. ${ }^{69}$ \\
\hline \multirow[t]{3}{*}{ Nimotuzumab } & HER1 & Cisplatino, $\mathrm{S}-1^{\dagger}$ & 5 & - & Wang, et al. ${ }^{70}$ \\
\hline & & Irinotecán ${ }^{\ddagger}$ & 2.4 & 8.3 & Satoh, et al. ${ }^{71}$ \\
\hline & & Docetaxel, cisplatino, 5FU ${ }^{\dagger \neq}$ & 6.5 & 12.5 & Xu, et al. ${ }^{72}$ \\
\hline Trastuzumab & HER2 & 5FU, capecitabina, cisplatino ${ }^{\dagger}$ & 6.7 & 13.8 & Bang, et al. ${ }^{29}$ \\
\hline \multicolumn{6}{|c|}{ Inhibidores de la tirosina cinasa } \\
\hline \multirow[t]{3}{*}{ Erlotinib } & HER1 & Solo $^{\dagger}$ & GC 1.6 & 3.5 & Dragovich, et al. ${ }^{73}$ \\
\hline & & & GEC 3 & 6.7 & \\
\hline & & Oxaliplatino, leucovorina, 5FU+ & 5.5 & 11 & Wainberg, et al. ${ }^{74}$ \\
\hline \multirow[t]{2}{*}{ Gefitinib } & HER1 & Solo $^{+}$ & - & 5.5 & Aldestein, et al. ${ }^{75}$ \\
\hline & & Cisplatino, 5FU, radioterapia ${ }^{\dagger}$ & - & 24.2 & Rodriguez, et al. ${ }^{76}$ \\
\hline \multirow[t]{4}{*}{ Lapatinib } & HER1,2 & Solo ${ }^{\dagger}$ & 2 & 4.8 & lqbal, et al. ${ }^{77}$ \\
\hline & & Capecitabina $^{\ddagger}$ & 1.6 & - & Lorenzen, et al. ${ }^{78}$ \\
\hline & & Capecitabina, oxaliplatino ${ }^{\dagger}$ & 6 & 12.2 & Hecht, et al. ${ }^{79}$ \\
\hline & & Paclitaxel $^{\ddagger}$ & 5.4 & 11 & Satoh, et al. ${ }^{80}$ \\
\hline Dacomitinib & HER1,2,4 & Solo & 2.1 & 7.1 & Lee, et al. ${ }^{81}$ \\
\hline
\end{tabular}


sobrevida libre de progresión (1.6-11 meses) en los pacientes con CG y gastroesophageal cancer (GEC), (Tabla 2); a pesar de esto, se tiene el registro de un paciente con CG metastásico tratado con cetuximab con la sobrevida libre de progresión más extensa reportada (7 años y 11 meses) ${ }^{82}$.

El panitumumab es el primer anticuerpo monoclonal IgG2 completamente humano aprobado para el tratamiento del cáncer colorrectal metastásico que expresa $E G F R$, basado en resultados que mostraron beneficios clínicos ${ }^{83}$. Un ensayo de fase III (REAL-3) evaluó el panitumumab en combinación con quimioterapia en pacientes con cáncer de esófago avanzado no tratado y CG, pero los resultados no mostraron un aumento de la sobrevida ${ }^{68}$ (Tabla 2). Otros cuatro ensayos clínicos fueron detenidos debido a la falta de eficacia del panitumumab adicional a la quimioterapia ${ }^{84}$. El fracaso de los estudios aleatorizados de fase III utilizando cetuximab o panitumumab en combinación con quimioterapia se debió principalmente a la no selección de una población específica de pacientes de acuerdo con la expresión de EGFR y a la interacción negativa del anticuerpo monoclonal EGFR y el agente quimioterapéutico. Estudios más recientes están evaluando la eficacia del panitumumab en conjunto con otros componentes ${ }^{84}$.

El matuzumab es un anticuerpo monoclonal lgG1 humanizado contra HER1. En un ensayo aleatorizado de fase II realizado en pacientes con CG avanzado con sobreexpresión de HER1 se evaluó matuzumab + epirubicina + cisplatino + capecitabina como tratamiento de primera línea, y los resultados mostraron que la combinación de matuzumab con quimioterapia no mejora las tasas de sobrevida (Tabla 2); por lo tanto, el matuzumab no fue probado en ensayos de fase $1 \mathrm{II}^{69,85}$.

El nimotuzumab es una IgG1 humanizada que ha demostrado eficacia sin toxicidad grave de la piel como la causada por otras terapias de unión a HER1; sin embargo, la sobrevida es similar a la observada con otros fármacos ${ }^{70-72}$ (Tabla 2).

\section{Terapias anti-HER2}

El trastuzumab es un anticuerpo monoclonal humanizado que se une al dominio extracelular IV de HER2 y previene la activación tirosina cinasa intracelular. Fue aprobado para su uso en combinación con quimioterapia como adyuvante para pacientes con cáncer de mama HER2+ y para terapia de CG avanzado en pacientes HER2+ no tratados previamente, en combinación con quimioterapia. Sin embargo, el trastuzumab confiere un mayor riesgo de cardiotoxicidad ${ }^{86}$, que es en gran parte reversible después de la eliminación del anticuerpo ${ }^{53,87}$. El único estudio que ha demostrado una fuerte evidencia para mejorar la supervivencia fue el ensayo de fase III ToGA, que incluyó pacientes con CG HER2+ (Tabla 2); los pacientes con tumores IHC 2+ y con hibridación in situ fluorescente positiva o tumores IHC 3+ tuvieron una marcada mejoría en la sobrevida (16.0 meses) ${ }^{30}$.

El pertuzumab es un anticuerpo monoclonal humanizado que se une al dominio de dimerización II de HER2, a diferencia del trastuzumab, que se une al dominio IV. Este inhibe la dimerización de HER2 con otros miembros de la familia HER y exhibe actividad antitumoral relacionada con la citotoxicidad celular dependiente de anticuerpo $(A D C C)^{88,89}$. Actualmente se investiga el efecto de añadir pertuzumab a la quimioterapia ${ }^{84}$.

Un metaanálisis sugirió que las terapias basadas en anticuerpos monoclonales anti-HER1 para pacientes con CG resultan en tasas de sobrevida menores que las terapias anti-HER2. Sin embargo, la quimioterapia junto con la terapia dirigida anti-HER2 incrementa significativamente el riesgo de presentar diarrea, hipocalcemia, mucositis y exantema en comparación con la quimioterapia sola ${ }^{85}$.

El trastuzumab emtansina (T-DM1) es un anticuerpo que combina trastuzumab y un inhibidor de microtúbulos derivado de maitansina (DM1). Este fármaco combina dos estrategias: la actividad anti-HER2 del trastuzumab y la entrega intracelular dirigida de DM1, que interfiere con la mitosis y promueve la apoptosis. Su empleo en el cáncer de mama HER2+ ya ha sido aprobado $^{90}$. Los ensayos clínicos están reclutando pacientes para evaluar el T-DM1 en conjunto con quimioterapia en pacientes con $\mathrm{CG}^{84,88,91}$.

\section{Terapias contra heterodímeros anti-HER2-HER3}

MM-111 es un anticuerpo de fusión biespecífico que comprende anticuerpos anti-HER2 y anti-HER3 unidos por albúmina sérica humana. MM-111 se une a las células que coexpresan HER2 y HER3, y bloquea la señalización inducida por el ligando ${ }^{92}$.

\section{Inhibidores de la tirosina cinasa}

\section{Inhibidores HER1}

El erlotinib ha sido aprobado para el tratamiento de los cánceres de pulmón y de páncreas ${ }^{54}$. En un 
ensayo de fase II se encontró que el erlotinib era activo en pacientes con cáncer gastroesofágico, pero no se obtuvo buena respuesta en pacientes con $\mathrm{CG}^{73}$ (Tabla 2). En un ensayo de fase II se administró una combinación de erlotinib, oxaliplatino, leucovorina y 5-fluorouracilo (5FU) a pacientes sin tratamiento previo con cáncer de esófago avanzado o metastásico y cáncer gastroesofágico; los resultados fueron similares a los reportadas previamente ${ }^{74}$ (Tabla 2).

El gefitinib fue aprobado por la FDA para el tratamiento del cáncer de pulmón de células no microcíticas avanzado ${ }^{54}$. En monoterapia se asocia con baja sobrevida ${ }^{75}$, pero en combinación con cisplatino, 5FU y radioterapia ha mostrado la mayor tasa de sobrevida (24.2 meses) en cáncer de esófago y gastroesofágico ${ }^{76}$ (Tabla 2).

El neratinib actúa uniéndose covalentemente a una cadena lateral de cisteína en el receptor HER1. Está dirigido a pacientes con mutaciones activantes en los genes EGFR, ERBB2 o ERBB3 y a pacientes con amplificación de EGFR.

\section{Inhibidores de HER1/HER2}

El lapatinib es una molécula oralmente activa de doble inhibición, para HER1 y HER2, que se ha autorizado para ser utilizada en el cáncer de mama HER2+. Es un inhibidor más potente para HER1 y HER2 que para HER4 (> 10x) ${ }^{91}$. Obtuvo los mejores resultados en pacientes con $C G$ avanzado con paclitaxel $^{71}$ y en combinación con capecitabina y oxaliplatino ${ }^{79}$, en comparación con su administración unica ${ }^{77}$ o combinada solo con capecitabina ${ }^{79}$ (Tabla 2).

El afatinib es un inhibidor irreversible de HER1 y HER2 que ha demostrado actividad antitumoral in vivo en pacientes con CG HER2 $+{ }^{91,93}$. Los ensayos clínicos están evaluando su eficacia en combinación con trastuzumab y con cisplatino más $5 \mathrm{FU}^{84}$.

\section{Paninhibidores (HER1/HER2/HER4)}

AST1306 es un inhibidor de HER oralmente activo, altamente selectivo e irreversible, con prometedora actividad anticáncer en pacientes con tumores sólidos avanzados ya tratados previamente. La respuesta parcial fue confirmada en el $12.7 \%$ de los pacientes (entre ellos, uno con CG) y se observó una enfermedad estable por $\geq 6$ meses en el $12.7 \%$ de pacientes. AST1306 puede inhibir potentemente la mutación EGFR T790M, la cual está comúnmente asociada con resistencia adquirida a los inhibidores HER1 de primera generación en el cáncer de pulmón de células no microcíticas ${ }^{94}$.

El dacomitinib induce apoptosis y detiene el ciclo celular en G1, e inhibe la fosforilación y la transducción de las vías de señalización en las células de CG HER2+. También bloquea la formación de heterodímeros HER1/HER2, HER2/HER3 y HER3/HER4. Se ha observado sinergia con muchos agentes citotóxicos comúnmente empleados (5FU, cisplatino, docetaxel y paclitaxel) y con agentes dirigidos, como el trastuzumab ${ }^{95}$. El dacomitinib fue empleado en pacientes con CG avanzado HER2+ como único agente tras el fallo de al menos un régimen de quimioterapia previa, pero no se observó una mejora sustancial de la sobrevida ${ }^{81}$ (Tabla 2).

El poziotinib inhibe la fosforilación de los miembros de la familia HER y moléculas de la cascada de señalización. También induce la apoptosis y el arresto en la fase del ciclo celular G1. Aunque este agente fue inactivo como monoterapia en líneas celulares HER2-, se reportó sinergia con quimioterapia en modelos tanto HER2+ como HER2-96.

AZD8931 proporciona inhibición de la señalización de EGFR, HER2 y HER3. La eficacia de este fármaco en combinación con paclitaxel fue probada en pacientes con CG avanzado; sin embargo, el ensayo se detuvo prematuramente debido a la baja respuesta observada ${ }^{84}$.

\section{Conclusiones}

La revisión realizada permite observar que existe una amplia heterogeneidad en las alteraciones moleculares como sobreexpresión y amplificación en el CG; las mutaciones somáticas no son comunes, ya que en todo el mundo solo se han reportado 88 diferentes mutaciones en los cuatro genes que codifican para las proteínas HER.

Mientras tanto, los tratamientos enfocados a mejorar la calidad de vida y prolongar la supervivencia en los pacientes con $\mathrm{CG}$ avanzado no han mostrado mejoras significativas en los últimos años; por lo tanto, se necesitan más estudios para identificar las mejores opciones para el tratamiento de estos pacientes. El hecho de que se hayan observado diferencias étnicas en los resultados de supervivencia en pacientes con otras malignidades en respuesta al tratamiento con inhibidores de la tirosina cinasa y quimioterapia (las cuales también se observaron en casos de CG) es de gran importancia, por lo cual debería ser tomado en cuenta. Afortunadamente, un mayor número de estudios está 
considerando actualmente el perfil molecular de los pacientes en la búsqueda de tratamientos, lo cual puede generar una mejor respuesta a los fármacos.

\section{Agradecimientos}

\author{
Agradecemos el apoyo en la revisión del manuscrito \\ a la Prof. Lourdes Carbajal Padilla.
}

\section{Bibliografía}

1. Ferlay J, Soerjomataram I, Ervik M, et al. GLOBOCAN 2012 v1.0. Cancer incidence and mortality worldwide: IARC CancerBase No. 11. Lyon, France: International Agency for Research on Cancer; 2013. (Consultado el 18 de febrero de 2016.) Disponible en: http://globocan. iarc.fr

2. Li H, Yu B, Li J, et al. Characterization of differentially expressed genes involved in pathways associated with gastric cancer. PLoS One. 2015;10:e0125013.

3. Roskoski R Jr. The ErbB/HER family of protein-tyrosine kinases and cancer. Pharmacol Res. 2014;79:34-74.

4. Gschwind A, Fischer OM, Ullrich A. The discovery of receptor tyrosine kinases: targets for cancer therapy. Nat Rev Cancer. 2004:4:361-70.

5. Hofmann M, Stoss O, Shi D, et al. Assessment of a HER2 scoring system for gastric cancer: results from a validation study. Histopathology. 2008;52:797-805.

6. Begnami MD, Fukuda E, Fregnani JH, et al. Prognostic implications of altered human epidermal growth factor receptors (HERs) in gastric carcinomas: HER2 and HER3 are predictors of poor outcome. J Clin Oncol. 2011;29:3030-6.

7. Jácome AA, Wohnrath DR, Scapulatempo Neto $C$, et al. Prognostic value of epidermal growth factor receptors in gastric cancer: a surviva analysis by Weibull model incorporating long-term survivors. Gastric Cancer. 2014; 17:76-86.

8. Aydin K, Okutur SK, Bozkurt M, et al. Effect of epidermal growth factor receptor status on the outcomes of patients with metastatic gastric cancer: a pilot study. Oncol Lett. 2014;7:255-9.

9. Braut T, Krstulja M, Kujundzić M, et al. Epidermal growth factor receptor protein expression and gene amplification in normal, hyperplastic, and cancerous glottic tissue: immunohistochemical and fluorescent in situ hybridization study on tissue microarrays. Croat Med J. 2009;50:370-9.

10. Inokuchi M, Murayama T, Hayashi M, et al. Prognostic value of co-expression of STAT3, mTOR and EGFR in gastric cancer. Exp Ther Med. 2011;2:251-6.

11. Terashima M, Kitada K, Ochiai A, et al. Impact of expression of human epidermal growth factor receptors EGFR and ERBB2 on survival in stage II/III gastric cancer. Clin Cancer Res. 2012;18:5992-6000.

12. Atmaca A, Werner D, Pauligk $C$, et al. The prognostic impact of epidermal growth factor receptor in patients with metastatic gastric cancer BMC Cancer. 2012;12:524.

13. Matsubara J, Yamada Y, Hirashima $Y$, et al. Impact of insulin-like growth factor type 1 receptor, epidermal growth factor receptor, and HER2 expressions on outcomes of patients with gastric cancer. Clin Cancer Res. 2008;14:3022-9.

14. Jørgensen JT, Hersom M. HER2 as a prognostic marker in gastric cancer - a systematic analysis of data from the literature. J Cancer. 2012;3:137-44

15. Choi MR, An $\mathrm{CH}$, Chung YJ, et al. Mutational and expressional analysis of ERBB3 gene in common solid cancers. APMIS. 2014;122:1207-12.

16. He XX, Ding L, Lin Y, et al. Protein expression of HER2, 3, 4 in gastric cancer: correlation with clinical features and survival. J Clin Pathol. 2015;68:374-80.

17. Yamashita K, Sakuramoto S, Watanabe M. Genomic and epigenetic profiles of gastric cancer: potential diagnostic and therapeutic applications. Surg Today. 2011;41:24-38.

18. Wu X, Chen Y, Li G, et al. Her3 is associated with poor survival of gastric adenocarcinoma: Her3 promotes proliferation, survival and migration of human gastric cancer mediated by PI3K/AKT signaling pathway. Med Oncol. 2014;31:903.

19. Hayashi M, Inokuchi M, Takagi $Y$, et al. High expression of HER3 is associated with a decreased survival in gastric cancer. Clin Cancer Res. 2008;14:7843-9.

20. Nielsen TO, Sorensen S, Dagnaes-Hansen F, et al. Directing HER4 mRNA expression towards the CYT2 isoform by antisense oligonucleotide decreases growth of breast cancer cells in vitro and in vivo. $\mathrm{Br} J$ Cancer. 2013;108:2291-8.
21. Kataoka $\mathrm{H}$, Joh $\mathrm{T}$, Kasugai $\mathrm{K}$, et al. Expression of mRNA for heregulin and its receptor, ErbB-3 and ErbB-4, in human upper gastrointestinal mucosa. Life Sci. 1998;63:553-64.

22. Wang YK, Gao CF, Yun T, et al. Assessment of ERBB2 and EGFR gene amplification and protein expression in gastric carcinoma by immunohistochemistry and fluorescence in situ hybridization. Mol Cytogenet. 2011;4:14.

23. Moutinho C, Mateus AR, Milanezi F, et al. Epidermal growth factor receptor structural alterations in gastric cancer. BMC Cancer. 2008;8:10.

24. Tsugawa K, Yonemura $\mathrm{Y}$, Hirono $\mathrm{Y}$, et al. Amplification of the c-met, c-erbB-2 and epidermal growth factor receptor gene in human gastric cancers: correlation to clinical features. Oncology. 1998;55:475-81.

25. Luber B, Deplazes J, Keller G, et al. Biomarker analysis of cetuximab plus oxaliplatin/leucovorin/5-fluorouracil in first-line metastatic gastric and oesophago-gastric junction cancer: results from a phase II trial of the Arbeitsgemeinschaft Internistische Onkologie (AIO). BMC Cancer. 2011;11:509.

26. Takehana T, Kunitomo K, Kono K, et al. Status of c-erbB-2 in gastric adenocarcinoma: a comparative study of immunohistochemistry, fluorescence in situ hybridization and enzyme-linked immuno-sorbent assay. Int J Cancer. 2002;98:833-7.

27. Tanner M, Hollmén M, Junttila TT, et al. Amplification of HER-2 in gastric carcinoma: association with topoisomerase Ilalpha gene amplification, intestinal type, poor prognosis and sensitivity to trastuzumab. Ann Oncol. 2005;16:273-8.

28. Park DI, Yun JW, Park JH, et al. HER-2/neu amplification is an independent prognostic factor in gastric cancer. Dig Dis Sci. 2006;51:1371-9.

29. Bang $\mathrm{Y}$, Chung $\mathrm{H}, \mathrm{Xu} \mathrm{J}$, et al. Pathological features of advanced gastric cancer (GC): relationship to human epidermal growth factor receptor 2 (HER2) positivity in the global screening programme of the ToGA trial. J Clin Oncol. 2009;27:15s.

30. Bang YJ, Van Cutsem E, Feyereislova A, et al. Trastuzumab in combination with chemotherapy versus chemotherapy alone for treatment of HER2-positive advanced gastric or gastro-oesophageal junction cancer (ToGA): a phase 3 , open-label, randomised controlled trial. Lancet. 2010;376:687-97.

31. Liu Z, Liu L, Li M, et al. Epidermal growth factor receptor mutation in gastric cancer. Pathology. 2011;43:234-8.

32. Abediankenari S, Jeivad F. Epidermal growth factor receptor gene polymorphisms and gastric cancer in Iran. Asian Pac J Cancer Prev. 2013;14:3187-90

33. Lee JW, Soung YH, Kim SY, et al. ERBB2 kinase domain mutation in a gastric cancer metastasis. APMIS. 2005;113:683-7.

34. Lee JW, Soung YH, Seo SH, et al. Somatic mutations of ERBB2 kinase domain in gastric, colorectal, and breast carcinomas. Clin Cancer Res. 2006;12:57-61.

35. Stephens $P$, Hunter $C$, Bignell G, et al. Lung cancer: intragenic ERBB2 kinase mutations in tumours. Nature. 2004;431:525-6.

36. Wang K, Kan J, Yuen ST, et al. Exome sequencing identifies frequent mutation of ARID1A in molecular subtypes of gastric cancer. Nat Genet. 2011;43:1219-23.

37. Soung YH, Lee JW, Kim SY, et al. Somatic mutations of the ERBB4 kinase domain in human cancers. Int J Cancer. 2006;118:1426-9.

38. Becker JC, Müller-Tidow C, Stolte M, et al. Acetylsalicylic acid enhances antiproliferative effects of the EGFR inhibitor gefitinib in the absence of activating mutations in gastric cancer. Int J Oncol. 2006;29:615-23.

39. Mammano $\mathrm{E}$, Belluco $\mathrm{C}$, Sciro $\mathrm{M}$, et al. Epidermal growth factor receptor (EGFR): mutational and protein expression analysis in gastric cancer. Anticancer Res. 2006;26:3547-50.

40. Mimori K, Nagahara H, Sudo T, et al. The epidermal growth factor receptor gene sequence is highly conserved in primary gastric cancers. J Surg Oncol. 2006;93:44-6.

41. $\mathrm{Xu} \mathrm{Z}, \mathrm{Huo} X, \mathrm{Ye} \mathrm{H}$, et al. Genetic mutation analysis of human gastric adenocarcinomas using ion torrent sequencing platform. PLoS One. 2014;9:e100442.

42. Forbes SA, Beare D, Gunasekaran P, et al. COSMIC: exploring the world's knowledge of somatic mutations in human cancer. Nucleic Acids Res. 2015;43(Database issue):D805-11.

43. Holbrook JD, Parker JS, Gallagher KT, et al. Deep sequencing of gastric carcinoma reveals somatic mutations relevant to personalized medicine. J Transl Med. 2011:9:119.

44. Jaiswal BS, Kljavin NM, Stawiski EW, et al. Oncogenic ERBB3 mutations in human cancers. Cancer Cell. 2013;23:603-17.

45. Bose R, Kavuri SM, Searleman AC, et al. Activating HER2 mutations in HER2 gene amplification negative breast cancer. Cancer Discov. 2013;3:224-37.

46. Yan M, Parker BA, Schwab R, et al. HER2 aberrations in cancer: implications for therapy. Cancer Treat Rev. 2014;40:770-80.

47. Lin WL, Kuo WH, Chen FL, et al. Identification of the coexisting HER2 gene amplification and novel mutations in the HER2 protein-overexpressed mucinous epithelial ovarian cancer. Ann Surg Oncol. 2011;18:2388-94.

48. Xu Z, Huo X, Ye H, et al. Genetic mutation analysis of human gastric adenocarcinomas using ion torrent sequencing platform. PLoS One. 2014;9:e100442. 
49. Martinelli E, De Palma R, Orditura M, et al. Anti-epidermal growth factor receptor monoclonal antibodies in cancer therapy. Clin Exp Immunol. 2009;158:1-9

50. Saltz LB, Lenz HJ, Kindler HL, et al. Randomized phase II trial of cetuximab, bevacizumab, and irinotecan compared with cetuximab and bevacizumab alone in irinotecan-refractory colorectal cancer: the BOND-2 study. J Clin Oncol. 2007;25:4557-61.

51. Vermorken JB, Mesia R, Rivera F, et al. Platinum-based chemotherapy plus cetuximab in head and neck cancer. N Engl J Med. 2008; 359:1116-27

52. Kim MA, Lee HJ, Yang HK, et al. Heterogeneous amplification of ERBB2 in primary lesions is responsible for the discordant ERBB2 status of primary and metastatic lesions in gastric carcinoma. Histopathology. 2011;59:822-31.

53. Morishita A, Gong J, Masaki T. Targeting receptor tyrosine kinases in gastric cancer. World J Gastroenterol. 2014;20:4536-45.

54. Kothari N, Almhanna K. Current status of novel agents in advanced gastroesophageal adenocarcinoma. J Gastrointest Oncol. 2015;6:60-74

55. Pinto $C$, Di Fabio $F$, Barone $C$, et al. Phase II study of cetuximab in combination with FOLFIRI in patients with untreated advanced gastric or gastroesophageal junction adenocarcinoma (FOLCETUX study). Ann Oncol. 2007:18:510-7.

56. Kanzler S, Trarbach T, Seufferlein T, et al. Cetuximab with irinotecan/ folinic acid/5FU as firstline treatment in advanced gastric cancer: a nonrandomized multicenter AIO phase II study. J Clin Oncol. 2009;27:15s.

57. Moehler M, Mueller A, Trarbach T, et al. Cetuximab with irinotecan, folinic acid and 5-fluorouracil as first-line treatment in advanced gastroesophageal cancer: a prospective multi-center biomarker-oriented phase II study. Ann Oncol. 2011;22:1358-66.

58. Yeh K, Hsu C, Hsu C, et al. Phase II study of cetuximab plus weekly cisplatin and 24 hour infusion of highdose 5fluorouracil and leucovorin for the firstline treatment of advanced gastric cancer. J Clin Oncol. 2009;27:15s

59. Han SW, Oh DY, Im SA, et al. Phase II study and biomarker analysis of cetuximab combined with modified FOLFOX6 in advanced gastric cancer. Br J Cancer. 2009;100:298-304.

60. Richards D, Kocs DM, Spira Al, et al. Results of docetaxel plus oxaliplatin $(D O C O X) \pm$ cetuximab in patients with metastatic gastric and/or gastroesophageal junction adenocarcinoma: results of a randomised phase 2 study. Eur J Cancer. 2013;49:2823-31.

61. Pinto C, Di Fabio F, Barone C, et al. Phase II study of cetuximab in combination with cisplatin and docetaxel in patients with untreated advanced gastric or gastro-oesophageal junction adenocarcinoma (DOCETUX study). Br J Cancer. 2009;101:1261-8.

62. Lordick F, Kang YK, Chung HC, et al. Capecitabine and cisplatin with or without cetuximab for patients with previously untreated advanced gastric cancer (EXPAND): a randomised, open-label phase 3 trial. Lancet Oncol. 2013;14:490-9

63. Li J, Liu X, Wang BY, et al. Phase II study of cetuximab in combination with modified FOLFIRI in patients with advanced gastric cancer who failed firstline chemotherapy (EFFI study). Clin Oncol. 2010;28:15s.

64. Schønnemann KR, Yilmaz M, Bjerregaard JK, et al. Phase II study of biweekly cetuximab in combination with irinotecan as second-line treatment in patients with platinum-resistant gastro-oesophageal cancer. Eur J Cancer. 2012;48:510-7.

65. Park SR, Kook MC, Choi IJ, et al. Predictive factors for the efficacy of cetuximab plus chemotherapy as salvage therapy in metastatic gastric cancer patients. Cancer Chemother Pharmacol. 2010;65:579-87.

66. Tebbutt NC, Parry MM, Zannino D, et al. Docetaxel plus cetuximab as second-line treatment for docetaxel-refractory oesophagogastric cancer: the AGITG ATTAX2 trial. Br J Cancer. 2013;108:771-4.

67. Chan JA, Blaszkowsky LS, Enzinger PC, et al. A multicenter phase II trial of single-agent cetuximab in advanced esophageal and gastric adenocarcinoma. Ann Oncol. 2011;22:1367-73.

68. Waddell T, Chau I, Cunningham D, et al. Epirubicin, oxaliplatin, and capecitabine with or without panitumumab for patients with previously untreated advanced oesophagogastric cancer (REAL3): a randomised open-label phase 3 trial. Lancet Oncol. 2013;14:481-9.

69. Rao S, Starling N, Cunningham D, et al. Matuzumab plus epirubicin cisplatin and capecitabine (ECX) compared with epirubicin, cisplatin and capecitabine alone as first-line treatment in patients with advanced oesophago-gastric cancer: a randomised, multicentre open-label phase II study. Ann Oncol. 2010;21:2213-9.

70. Wang JW, Chi Y, Zheng ZX, et al. Randomized, singlecentered, phase II clinical trial of nimotuzumab plus cisplatin and S1 as first line therapy in patients with advanced gastric cancer. J Clin Oncol. 2012;30(Suppl):e14668.

71. Satoh T, Lee KH, Rha SY, et al. Randomized phase II trial of nimotuzumab plus irinotecan versus irinotecan alone as second-line therapy for patients with advanced gastric cancer. Gastric Cancer. 2015;18;824-32.

72. Xu CD. Clinical study of nimotuzumab combined with chemotherapy in the treatment of late stage gastric cancer. Asian Pac J Cancer Prev. 2014;15:10273-6
73. Dragovich T, McCoy S, Fenoglio-Preiser CM, et al. Phase II trial of erlotinib in gastroesophageal junction and gastric adenocarcinomas: SWOG 0127. J Clin Oncol. 2006;24:4922-7.

74. Wainberg ZA, Lin LS, DiCarlo B, et al. Phase II trial of modified FOLFOX6 and erlotinib in patients with metastatic or advanced adenocarcinoma of the oesophagus and gastro-oesophageal junction. $\mathrm{Br} \mathrm{J}$ Cancer. 2011;105:760-5.

75. Adelstein DJ, Rodríguez CP, Rybicki LA, et al. A phase II trial of gefitinib for recurrent or metastatic cancer of the esophagus or gastroesophageal junction. Invest New Drugs. 2012;30:1684-9.

76. Rodríguez CP, Adelstein DJ, Rice TW, et al. A phase II study of perioperative concurrent chemotherapy, gefitinib, and hyperfractionated radiation followed by maintenance gefitinib in locoregionally advanced esophagus and gastroesophageal junction cancer. J Thorac Oncol. 2010:5:229-35.

77. Iqbal S, Goldman B, Fenoglio-Preiser CM, et al. Southwest Oncology Group study S0413: a phase II trial of lapatinib (GW572016) as first-line therapy in patients with advanced or metastatic gastric cancer. Ann Oncol. 2011;22:2610-5.

78. Lorenzen S, Riera Knorrenschild J, Haag GM, et al. Lapatinib versus lapatinib plus capecitabine as second-line treatment in human epidermal growth factor receptor 2-amplified metastatic gastro-oesophageal cancer: a randomised phase II trial of the Arbeitsgemeinschaft Internistische Onkologie. Eur J Cancer. 2015;51:569-76.

79. Hecht JR, Bang YJ, Qin S, et al. Lapatinib in combination with capecitabine plus oxaliplatin (CapeOx) in HER2-positive advanced or metastatic gastric, esophageal, or gastroesophageal adenocarcinoma (AC): the TRIO-013/LOGiC trial. J Clin Oncol. 2013;31(Suppl):LBA4001.

80. Satoh $\mathrm{T}, \mathrm{Xu} \mathrm{RH}$, Chung $\mathrm{HC}$, et al. Lapatinib plus paclitaxel versus paclitaxel alone in the second-line treatment of HER2-amplified advanced gastric cancer in Asian populations: TyTAN - a randomized, phase III study. J Clin Oncol. 2014;32:2039-49.

81. Lee KH, Oh DY, Lee KW, et al. A phase II open label trial of PF-00299804 monotherapy in patients with HER-2 positive advanced gastric cancer after failure of at least one prior chemotherapy regimen. Ann Oncol. 2012;23(Suppl 11):xi19-20.

82. Adua D, Di Fabio F, Rojas Llimpe FL, et al. Long-term survival in an advanced gastric cancer patient treated with cetuximab in association with FOLFIRI: a case report. J Gastrointest Oncol. 2014:5:E13-7.

83. Gibson TB, Ranganathan A, Grothey A. Randomized phase III trial results of panitumumab, a fully human anti-epidermal growth factor receptor monoclonal antibody, in metastatic colorectal cancer. Clin Colorectal Cancer. 2006;6:29-31.

84. ClinicalTrials.gov. Bethesda (MD): National Library of Medicine (US). 2000-2015. (Consultado el 5 de julio de 2016.) Disponible en: https:// clinicaltrials.gov

85. Luo $\mathrm{HQ}$, Han L, Jiang Y. Meta-analysis of six randomized control trials of chemotherapy plus anti-HER monoclonal antibody for advanced gastric and gastroesophageal cancer. Asian Pac $\mathrm{J}$ Cancer Prev. 2014;15:5343-8.

86. Seidman A, Hudis C, Pierri MK, et al. Cardiac dysfunction in the trastuzumab clinical trials experience. J Clin Oncol. 2002;20:1215-21.

87. Ewer MS, Vooletich MT, Durand JB, et al. Reversibility of trastuzumab-related cardiotoxicity: new insights based on clinical course and response to medical treatment. J Clin Oncol. 2005;23:7820-6.

88. Ayyappan S, Prabhakar D, Sharma N. Epidermal growth factor receptor (EGFR)-targeted therapies in esophagogastric cancer. Anticancer Res. 2013;33:4139-55.

89. Franklin MC, Carey KD, Vajdos FF, et al. Insights into ErbB signaling from the structure of the ErbB2-pertuzumab complex. Cancer Cell. 2004;5:317-28.

90. Ballantyne A, Dhillon S. Trastuzumab emtansine: first global approval. Drugs. 2013;73:755-65.

91. Moorcraft SY, Chau I. Investigational therapies targeting the ErbB family in oesophagogastric cancer. Expert Opin Investig Drugs. 2014; 23:1349-63.

92. McDonagh CF, Huhalov A, Harms BD, et al. Antitumor activity of a novel bispecific antibody that targets the ErbB2/ErbB3 oncogenic unit and inhibits heregulin-induced activation of ErbB3. Mol Cancer Ther. 2012:11:582-93.

93. Janjigian $\mathrm{YY}$, Viola-Villegas $\mathrm{N}$, Holland JP, et al. Monitoring afatinib treatment in HER2-positive gastric cancer with 18F-FDG and 89Zr-trastuzumab PET. J Nucl Med. 2013:54:936-43.

94. Zhang J, Cao J, Li J, et al. A phase I study of AST1306, a novel irreversible EGFR and HER2 kinase inhibitor, in patients with advanced solid tumors. J Hematol Oncol. 2014;7:22.

95. Nam HJ, Ching KA, Kan J, et al. Evaluation of the antitumor effects and mechanisms of PF00299804, a pan-HER inhibitor, alone or in combination with chemotherapy or targeted agents in gastric cancer. Mol Cancer Ther. 2012;11:439-51.

96. Nam HJ, Kim HP, Yoon YK, et al. Antitumor activity of HM781-36B, an irreversible Pan-HER inhibitor, alone or in combination with cytotoxic chemotherapeutic agents in gastric cancer. Cancer Lett. 2011;302:155-65. 